\title{
Acromyrmex Mayr (Hymenoptera: Formicidae: Myrmicinae): patrones de distribución de las especies en la provincia de Buenos Aires, Argentina
}

\author{
Susana CULEBRA MASON ${ }^{1}$, Carolina SGARBI ${ }^{2}$, Jimena CHILA COVACHINA ${ }^{2 *}$, Juan Martín \\ PEÑA $^{2}$, Nadia DUBROVSKY BERENSZTEIN ${ }^{3}$, Cecilia MARGARÍA ${ }^{1,4}$ \& Mónica RICCI ${ }^{1,2}$
}

${ }^{1}$ Zoología Agrícola, Facultad de Ciencias Agrarias y Forestales, Universidad Nacional de La Plata, 60 y 118, (1900) La Plata, Buenos Aires, Argentina. ${ }^{2}$ Zoología Agrícola, Escuela de Ciencias Agrarias, Ambientales y Naturales, Universidad Nacional del Noroeste de la provincia de Buenos Aires, Roque Sáenz Peña 456, (6000) Junín, Buenos Aires, Argentina. ${ }^{3}$ Agroecología, Facultad de Ciencias Agrarias y Forestales, Universidad Nacional de La Plata, 60 y 118, (1900) La Plata, Buenos Aires, Argentina. ${ }^{4}$ División Entomología, Facultad de Ciencias Naturales y Museo, Universidad Nacional de La Plata, Paseo del Bosque sin número, (1900) La Plata, Buenos Aires, Argentina. *Becaria de la Comisión de Investigaciones Científicas de la provincia de Buenos Aires

\begin{abstract}
Acromyrmex Mayr (Hymenoptera: Formicidae: Myrmicinae): species distribution patterns in the province of Buenos Aires, Argentina. The Attini, mainly the genera Atta Fabricius and Acromyrmex Mayr, are called leaf-cutting ants. Acromyrmex has scarce records referring to the geographical distribution of its species in Buenos Aires province. The objective of the present work was to gather existing information, to update these data with our own investigation, and to apply different criteria to establish relations with the use of the land. Of the total records for the province, 87 localities of 56 provincial districts were counted. To perform a hierarchical classification of similarities in the distribution patterns of the species, binary data matrices were constructed, analyzed by the weighted average method, calculating squared euclidean distances. The cophenetic indexes obtained were 0.883 for the geographical subregions, 0.943 for the municipal conglomerates and 0.877 for the agricultural areas. Eleven species of ants of the genus Acromyrmex are present in the province of Buenos Aires: A. ambiguus, A. fracticornis, A. heyeri, A. hispidus, A. hystrix, A. landolti, A. lobicornis, A. lundii, A. rugosus, A. silvestrii and A. striatus. The genus Acromyrmex is characterized by nesting in the soil, whereby anthropic practices for productive, industrial and urban purposes directly affect its pattern of occurrence.
\end{abstract}

Key words: formicids, myrmicines, geographical distribution, leaf-cutter ants

Resumen: Las Attini, representadas principalmente por los géneros Atta Fabricius y Acromyrmex Mayr, son llamadas hormigas cortadoras. Acromyrmex cuenta con escasos registros referidos a la distribución geográfica de sus especies en la provincia de Buenos Aires. El objetivo del presente trabajo fue reunir los datos existentes, actualizar con aportes propios la distribución y aplicar distintos criterios que permitan establecer relaciones con el uso de la tierra. Del total de registros para la provincia, se contabilizaron 87 localidades de 56 partidos. Para realizar una clasificación jerárquica de similitudes en los patrones de distribución de las especies, se construyeron matrices de datos binarios, analizadas por el método del promedio ponderado calculando las distancias euclídeas al cuadrado. Los índices cofenéticos obtenidos fueron 0.883 para las subregiones geográficas, 0.943 para los conglomerados municipales y 0.877 para las zonas agropecuarias. Para la provincia de Buenos Aires se han registrado 11 especies de hormigas del género Acromyrmex: A. ambiguus, A. fracticornis, A. heyeri, A. hispidus, A. histrix, A. landolti, A. lobicornis, A. lundii, A. rugosus. A. silvestrii y A. striatus. El género Acromyrmex se caracteriza por nidificar en el suelo, por lo cual las prácticas que realiza el hombre con fines productivos, industriales y urbanísticos afectan en forma directa su patrón de ocurrencia.

Palabras clave: formícidos, mirmicinos, distribución geográfica, hormigas podadoras

\section{INTRODUCCIÓN}

Las hormigas (Hymenoptera: Formicidae) constituyen uno de los grupos más abundantes y diversos de insectos a nivel mundial (Hölldobler \& Wilson, 1990, 2011), asignadas a 17 subfami- lias, 334 géneros, 13.258 especies y 1.946 subespecies (Bolton, 2016); mientras que otras referencias indican 21 subfamilias, 491 géneros y 24.614 especies/subespecies (Antweb, 2017). Son consideradas de importancia tanto en los sistemas naturales como en los modificados por el hombre 
(Majer, 1983), al mismo tiempo que participan en todos los niveles tróficos (Folgarait, 1998, Harada, 2016). Para la Región Neotropical están representadas 4.565 especies, pertenecientes a 14 subfamilias y 173 géneros vivientes (Fernández \& Sendoya, 2004; Antweb, 2016). La República Argentina, por su ubicación geográfica, ofrece una amplia gama de nichos susceptibles de ser ocupados por las hormigas, favoreciendo así su diversidad específica (Cuezzo, 1998). Así se reconocen 11 subfamilias, 82 géneros y 629 especies/ subespecies (Antweb, 2016).

Las hormigas cortadoras de hojas son consideradas los herbívoros dominantes del Neotrópico ya que consumen mucha más vegetación que cualquier otro grupo de animales de diversidad taxonómica comparable (Höldobler \& Wilson, 1990). Recientes estudios evolutivos de mirmicinos referidos a la filogenia y biogeogeografía (Ward et al., 2015) establecen numerosos cambios nomenclaturales, especialmente a nivel tribal: Attini (=Pheidolini Emery, Dacetini Forel, Orectognathina Brown, Ochetomyrmecini Emery, Cephalotini M.R. Smith, Basicerotini Brown, Phalacromyrmecini Dlussky \& Fedoseeva, Blepharidattini Wheeler \& Wheeler y Lenomyrmecini Bolton) está conformado por 46 géneros con otros regímenes alimentarios además de los micetófagos, como son las hormigas cortadoras. En el presente estudio nos referiremos a la Tribu Attini en el sentido tradicional, en el cual las hormigas cortadoras son consideradas las "Attini superiores". Están distribuidas predominantemente en la Región Neotropical, con más de 200 especies reunidas en 13 géneros (De Souza et al., 2007; Culebra Mason et al., 2009). Se encuentran ampliamente distribuidas en casi todo el territorio, desde el Sur de Norteamérica hasta la Patagonia, ocupando una gran variedad de ambientes (selvas, pastizales, bosques, estepas), a excepción de la costa oeste de Chile y Perú (Farji-Brener \& Ruggiero, 1994). Viven en hormigueros subterráneos y poseen organización social (Anglada et al., 2013), y establecen simbiosis con hongos de la familia Agariceae: Basidiomycota) del cual se alimentan (De Souza et al., 2007; Culebra Mason et al., 2009).

Las "Attini inferiores" comprenden ocho géneros, muy poco conspicuos, que no atacan directamente a las plantas y usan heces de insectos y material en descomposición como sustrato para el hongo. Los restantes cinco géneros constituyen el grupo de las "Attini superiores", representadas principalmente por dos géneros: Atta Fabricius y Acromyrmex Mayr, las más común- mente llamadas hormigas cortadoras (Nobua Behrmann, 2014). Su polifagia, abundancia, capacidad de atacar plantas nativas y habilidad de prosperar consumiendo algunas pocas especies vegetales, las convierte en plagas (Cherrett et al., 1989; Lugo et al., 2013). En la República Argentina, Atta ha sido registrada en las provincias de Misiones, Corrientes, Santa Fe, Salta, Jujuy, Tucumán y Formosa (De Coll, inéd.; Vittar \& Cuezzo, 2008). Cuezzo (1998) menciona 18 especies y 10 subespecies de Acromyrmex, en la página Antmaps (Janicki et al., 2016) menciona 22 especies y 10 subespecies, y Antweb (2016) 13 especies y 8 subespecies; siendo escasos los trabajos referidos a la distribución geográfica de las mismas, más aún a nivel provincial (Farji- Brener \& Ruggiero, 1994; Ricci et al., 2005).

Desde el punto de vista biogeográfico (Morrone, 2001), la provincia de Buenos Aires pertenece a la Región Neotropical, subregión Chaqueña, Provincia de la Pampa. La vegetación está representada por sabanas de gramíneas que pueden alcanzar hasta $1 \mathrm{~m}$ de altura, hierbas y arbustos; bosques xéricos empobrecidos; sabanas inundables; y bosques en galería a lo largo de los ríos. En las sabanas, las especies dominantes de plantas pertenecen a los géneros Aristida L., Bothriochloa Kuntze, Briza L., Bromus L., Eragrostis Wolf, Melica L., Panicum L., Paspalum L., Piptochaetium J. Presl, Schizachyrium Nees y Stipa L. Entre las especies dominantes de plantas se encuentran Acacia caven (Molina) Molina, Allophylus edulis (A.St.-Hil.) Radlk. ex Warm, Andropogon lateralis Nees, Aspidosperma quebracho-blanco Schltdl, Butia yatay Becc., Celtis iguanaea (Jacq.) Sarg., Cyclolepis genistoides Don, Elionurus muticus (Spreg.) Kuntze, Zanthoxylum fagara (L.) Sarg., Geoffroea decorticans Burkart, Heterostachys ritteriana (Moq.) Ung.-Sternb., Panicum racemosum Spreng., Paspalum almum Chase, $P$. notatum Flüggé , Prosopis alba Grisebach., P. affinis Sprengel, $P$. caldenia Burkart, $P$. flexuosa De Candolle, $P$. nigra (Grisebach) Hieronymus y Schinus longifolius (Lindl.) Speg. La provincia de la Pampa se halla amenazada por la conversión de hábitats naturales para la agricultura, la degradación de los suelos por el sobrepastoreo, la canalización y drenaje de cuerpos de agua naturales y la introducción de especies exóticas (Morrone, 2001).

El uso de la tierra con fines urbanísticos, industriales y agropecuarios ocasiona pérdida de la diversidad biológica, especialmente de aquellos organismos que nidifican en el suelo como las hormigas. Por ello, el conocimiento de su diver- 
sidad permite inferir el disturbio actual de los ecosistemas (Morello et al., 2000). Las modificaciones del hábitat, como producto de disturbios naturales o antropogénicos, pueden tener considerables efectos en la composición de la comunidad de hormigas en términos de diversidad de especies y organización (Bestelmeyer \& Wiens, 1996; Verzero Villalba et al., 2014).

Por lo expuesto y por la escasa información sobre las especies de hormigas cortadoras del género Acromyrmex presentes en la provincia de Buenos Aires, se plantearon los siguientes objetivos:

Reunir los datos existentes y actualizar con aportes propios los registros referidos a la distribución.

Aplicar distintos criterios de clasificación de regiones y zonas productivas que permitan relacionar patrones de distribución de las especies con el uso de la tierra para diferentes fines.

\section{MATERIALES Y MÉTODOS}

\section{Recolección e identificación del material}

Se realizó un relevamiento faunístico de las especies del género Acromyrmex en la provincia de Buenos Aires, Argentina. El mismo se llevó a cabo sobre datos de recolección de material de hormigas cortadoras realizado por el equipo de investigación desde 1980 hasta 2015 publicadas e inéditas; además se completó con información citada a la fecha (Ver referencias en el Apéndice). Cabe destacar que aquéllas publicaciones que no explicitaran la localidad de recolección de las cortadoras no fueron tenidas en cuenta. Asimismo, se revisó el material de formícidos depositado en el Museo de La Plata, Buenos Aires, Argentina.

Para la toma de muestras se utilizaron trampas de caída tipo Pitfall y captura directa con pincel (Agosti et al., 2000; Blondel et al., 2010; Verzero et al., 2014) en 54 localidades bonaerenses de 36 partidos. El material fue identificado mediante el uso de claves taxonómicas (Kusnezov, 1978; Gonçalves, 1961), e identificado bajo microscopio binocular estereoscópico. Los "voucher specimens" están depositados en el Curso de Zoología Agrícola de la Facultad de Ciencias Agrarias y Forestales de la Universidad Nacional de La Plata, Argentina.

\section{Criterios de agrupamiento aplicados}

Dado que desde el punto de vista biogeográfico la provincia de Buenos Aires es uniforme (provincia de la Pampa -Morrone, 2001-), y dadas las transformaciones actuales que han sufrido algu- nas regiones por la expansión de las superficies cultivadas hacia las zonas urbanas, periurbanas e industriales, se decidió tomar otros criterios de clasificación que permitieran estudiar las especies de hormigas: a) subregiones geográficas (SACyP, 1995), b) conglomerados municipales (Quesada Aramburú \& Cadelli, 2012), y c) zonas agropecuarias (Quesada Aramburú \& Cadelli, 2012).

Subregiones geográficas: Considera las diferencias climáticas, edáficas y fisiográficas heterogéneas, variedad de usos y sistemas productivos, reconociendo siete subregiones geográficas: Pampa arenosa (clima templado subhúmedo con época de invierno seca, llanura con pendiente suave O-E y suelos franco-arenosos a arenosos, con poca materia orgánica), Pampa ondulada (clima subhúmedo con precipitaciones medias anuales de $900 \mathrm{~mm}$, temperaturas extremas entre $9^{\circ} \mathrm{C}$ en julio y $41,5^{\circ} \mathrm{C}$ en enero; pérdida de estabilidad estructural en el horizonte de labranza que se transforma en factor de degradación de los suelos de esta región por el proceso de agriculturización), Tandilia (clima subhúmedohúmedo, con precipitaciones medias anuales de $800 \mathrm{~mm}$ y las temperaturas extremas de $7,1^{\circ} \mathrm{C}$ en julio y $28,6^{\circ} \mathrm{C}$ en enero; cadena de sierras con alturas que no superan los 500 metros s.n.m.) y Ventania (ubicada más al oeste; clima algo más seco, que Tandilia, régimen subhúmedo-seco, con precipitaciones anuales medias que oscilan entre 700 y $750 \mathrm{~mm}$; temperaturas extremas bajas con mínima absoluta de $9,9^{\circ} \mathrm{C}$ y máxima absoluta de $41,2^{\circ} \mathrm{C}$; prevalecen los suelos medianamente profundos, aptos para el laboreo); Pampa Deprimida (llanura plana que abarca prácticamente toda la cuenca del Río Salado y la del Arroyo Vallimanca; régimen pluviométrico anual que oscila de $1000 \mathrm{~mm}$ y $800 \mathrm{~mm}$, existencia de una pendiente de muy bajo gradiente regional y la falta de una red de drenaje definida; suelos con horizonte superficial somero y, más en profundidad, poseen elevados contenidos de arcilla; grandes sectores con una capa compacta de costra calcárea a escasa profundidad aptos para pastizal natural que satisface las necesidades de cría de ganado), Interserrana (llanura loésica entre los sistemas serranos de Tandilia y Ventania con drenaje suave hacia el océano Atlántico, clima subhúmedo-seco, con precipitaciones anuales superiores a $700 \mathrm{~mm}$, decreciendo de $\mathrm{E}$ a O, temperatura media anual de $14^{\circ} \mathrm{C}$; actividad productiva mixta agrícola-ganadera -trigo, avena y cebada, sorgo granífero y girasol-, Delta del Paraná (constituida por sedimentos fluvia- 
les aportados por el río Paraná, paisaje insular de gran extensión, con brazos de río y cauces abandonados, colmados por materiales finos; suelos con limitaciones diversas: escaso desarrollo, anegabilidad, presencia de capa freática habitual, salinidad y alcalinidad), y Sector Patagónico (rasgos de transición entre el sistema serrano hacia la zona patagónica, clima subhúmedo-seco en la parte $\mathrm{N}$, semiárido hacia el S, precipitaciones medias anuales entre 600 y $400 \mathrm{~mm}$, llanura ondulada septentrional y terrazas aluvionales de origen marino hacia el S, suelos de depósitos de arena en forma de delgada cobertura dando perfiles sumamente someros.

Conglomerados municipales: Se basa en el análisis de conglomerados o "clusters" para clasificar a los municipios en seis grupos relativamente homogéneos (indicadores de índole geográfica, demográfica, social y productiva): 1) industrial, que rodea a la Ciudad Autónoma de Buenos Aires (mayoritariamente con actividades manufactureras, teniendo el sector primario y el turístico una mínima expresión); 2) industrial mixto, con mayores carencias económicas, que rodea al primero y además incluye algunas ciudades del interior (si bien la actividad predominante es la industrial, se observa cierta presencia del sector primario, principalmente con ganadería y algunos destinos turísticos; aunque también se destinan algunas tierras al cultivo de soja y trigo); 3) ganadero, que abarca la región central y ciertos municipios del extremo sur (principalmente establecimientos dedicados a cría de ganado bovino); 4) oleaginoso, ubicado al norte extendiéndose hacia el oeste (constituyendo la región agrícola más importante del país, siendo el cultivo de soja el de mayor difusión, esta zona se caracteriza por la aptitud de sus suelos y condiciones climáticas normalmente favorables); 5) cerealero, emplazado en el sur (destacándose el cultivo de trigo por ser el más difundido, si bien también son relevantes el cultivo de soja y la actividad ganadera); y 6) turístico, localizado sobre la costa atlántica (este conglomerado carece de actividades primarias o industriales de relevancia).

Zonas agropecuarias: Considera los recursos naturales y características del sector agropecuario teniendo en cuenta la zonificación aportada por Slutzky (1968) hace varias décadas pero aun considerada en clasificaciones más recientes (Lazzarini et al., 2004; Cappello \& Fortunato, 2008), en las cuales se reconocen las siguientes zonas agropecuarias: Zona hortícola del GBA (I), Zona tambera (II), Zona agrícola del Norte (III), Zona de invernada (IV), Zona de cría (V) y Zona agrícola del Sur (VI).

(I) Zona hortícola del GBA: el área se caracteriza por ser periurbana y presentar límites imprecisos entre la ciudad y el campo. La superficie efectiva agropecuaria es de 214.300 ha de las cuales el $25 \%$ tiene aptitud agrícola-ganadera, el 55\% ganadera-agrícola y el $20 \%$ ganadera. Dentro de las actividades agropecuarias, la horticultura y la floricultura son las más importantes, destacándose la producción de hortalizas que se realiza a campo y bajo cubierta. (II) Zona tambera: predominan los ambientes caracterizados por extensas planicies y depresiones, con suelos con alto contenido de sales, poco profundos y anegables (55\% de la superficie), paisajes de lomadas con planicies y depresiones aisladas (30\%) y relieves con características de pampa ondulada (15\%). El 67,4\% de los suelos tiene aptitud ganadera, el $23,5 \%$ aptitud agrícola-ganadera y solo el $2,5 \%$ restante de la superficie posee aptitud agrícola. (III) Zona agrícola del Norte: a) subzona núcleo agrícola, presenta $42 \%$ con aptitud agrícola, siendo el principal cultivo la soja seguida por trigo y maíz; y b) subzona mixta del centro, posee una gran proporción de suelos con aptitud mixta $(80 \%)$ siendo el resto de aptitud exclusivamente ganadera $(12 \%)$ y el resto agrícola (8\%). (IV) Zona de invernada: posee una alta proporción de suelos con capacidad de uso agrícola-ganadero. Aproximadamente el $70 \%$ del área se destina a la ganadería (recría e invernada), y el resto a la agricultura siendo los cultivos más importantes girasol maíz y en menor medida soja, trigo y sorgo. (V) Zona de cría: en el $90 \%$ de la superficie de esta zona solo pueden realizarse actividades agrícolas circunstanciales, por lo que las alternativas de combinación de actividades resultan difíciles y la actividad ganadera aparece como la opción dominante. La agricultura se realiza en los mejores suelos -las lomadas, áreas de poca extensión-. Los principales cultivos son trigo, girasol y maíz. Tanto en los sistemas ganaderos de cría, en los de cría y recría y en los de ciclo completo (explotaciones que superan las 200 ha), entre el 70 y 90\% de la superficie está ocupada por campo natural y entre el $10-15 \%$ por pasturas. En los sistemas combinados ganadería (70\%)-agricultura (30\%), explotaciones de entre 300 y 500 ha, el $40 \%$ de la superficie ganadera está ocupada por campo natural y el $60 \%$ por pasturas y cultivos como suplemento a la ganadería. (VI) Zona agrícola del Sur: esta región es muy diversa; hacia el sureste predominan los suelos agrícolas o agrícolas-ganaderos en un $91 \%$, el restante 9\% son suelos no arables. En cuanto al 
uso del suelo, el $50 \%$ se dedica a la agricultura y otro tanto a la ganadería; mientras que hacia el suroeste de la misma, el $58 \%$ de los suelos tiene aptitud ganadero-agrícola, admite rotación, pero la fase agrícola (girasol-maíz) es corta, luego de un largo período de pasturas perennes. El $28 \%$ de los suelos tiene aptitud agrícola-ganadera y el restante $14 \%$ aptitud ganadera. Esta zona soporta condiciones hídricas desfavorables, mientras que hacia el sur de la provincia de Buenos Aires se encuentra una zona de riego (Valle inferior del Rio Colorado) con establecimientos de menos de 100 ha, caracterizados por la realización de actividades hortícolas intensivas (cebolla-zapallo-tomate), establecimientos de 100-1000 ha con sistemas agrícolas (cereales y forrajes)- ganaderos y establecimientos de 500-100 ha cuya principal actividad es la ganadería sobre pastizales naturales, verdeos y pasturas y una zona de secano (partido de Carmen de Patagones), caracterizada por sistemas mixtos-ganaderos, entre 70 y $80 \%$ de la superficie dedicada a ganadería. Dentro de la actividad agrícola predominan cultivos de cosecha fina.

\section{Análisis estadístico}

Para encontrar la existencia de similitudes en los patrones de distribución de las especies, se construyó una matriz de datos binarios (presencia/ausencia de las especies por conglomerados), a los fines de realizar una clasificación jerárquica de las especies mediante el método del promedio ponderado (Johnson, 1967), calculando las distancias euclídeas al cuadrado. Se utilizó el software estadístico InfoStat (Di Rienzo et al., 2008).

Para obtener una clasificación jerárquica de las especies en función de sus distribuciones, se partió de una matriz de datos binarios (presencia/ ausencia de las especies por subregión geográfica-conglomerado municipal- zona agropecuaria), se aplicó el coeficiente de similitud de distancia "Simple Matching" (Sokal \& Michener, 1958) y el método de encadenamiento por promedios ponderados del Análisis de Conglomerados (Weighted Pair Group Method Average, WPGMA) (Sokal \& Michener, 1958; Mc Quitty, 1966).

\section{RESULTADOS}

Del total de especies de hormigas del género Acromyrmex citadas para nuestro país, en el presente estudio se reconocen 11 de ellas, distribuidas en 56 partidos de la provincia de Buenos Aires, 34 de los cuales constituyen ampliaciones de su distribución: A. ambiguus (Emery), A. fracticornis (Forel), A. heyeri (Forel), A. hispidus Santschi, A. hystrix (Latreille), A. landolti (Forel), A. lobicornis (Emery), A. lundii (GuérinMéneville), A. rugosus (Smith), A. silvestrii (Emery) y A. striatus (Roger) (Apéndice, Fig. 1). Cabe mencionar que A. subterraneus (Forel) ha sido citada a nivel provincial, pero sin especificar localidad o partido.

El análisis de los resultados obtenidos al considerar la distribución de las especies de Acromyrmex en las subregiones geográficas se muestra en la Figura 2. Se presentan dos grandes grupos de especies: (A. hispidus + lundi + lobicornis + ambiguus) y (A. strriatus + silvestrii + landolti + fracticornis + heyeri + rugosus + hystrix), con un índice de correlación cofenética de 0.883. La subregión Pampa arenosa incluye al mayor número de especies (siete), le sigue en cantidad decreciente la Pampa ondulada (seis), Tandilia y Ventania (cinco), Sector patagónico (cuatro), Pampa deprimida y Pampa entre montañas (tres cada una), y finalmente Metropolitana ondulada y Delta (dos). Las especies A. rugosus e hystrix se encontraron únicamente en Pampa ondulada, mientras que $A$. fracticornis + landol$t i$ sólo en Pampa arenosa. Se destaca que la única especie presente en todas las subregiones fue $A$. lundii (Apéndice).

Los resultados del análisis estadístico aplicado sobre la base de los conglomerados municipales (índice de correlación cofenética $=0.943$ ) se muestran en la Figura 3. Se destaca la formación de dos grandes grupos de especies: (A. hispidus + striatus + lundii + lobicornis + ambiguus) y (A. silvestrii + rugosus + landolti + fracticornis + heyeri + hystrix). El conglomerado oleaginoso incluye el mayor número de especies (a excepción de A. striatus, silvestrii e hystrix) y en orden decreciente continúan el industrial mixto (siete especies), el ganadero (seis especies), el industrial, cerealero y turístico (tres especies cada uno) (Fig. 1). En el conglomerado industrial se destaca la presencia de A. lundii+striatus+hispidus y A. landolti+fracticornis +rugosus son exclusivas del oleaginoso.

Los resultados del análisis estadístico aplicado sobre la base de las zonas agropecuarias (índice de correlación cofenética $=0.877$ ) $\mathrm{se}$ muestran en la Figura 4. Se forman dos grupos de especies. Uno de ellos lo componen el núcleo de especies $A$. lundii + ambiguus con A. hispidus; en el otro, se forman el grupo fracticornis + landolti y A. heyeri + lobicornis + striatus + rugosus + silvestrii + hystrix. 


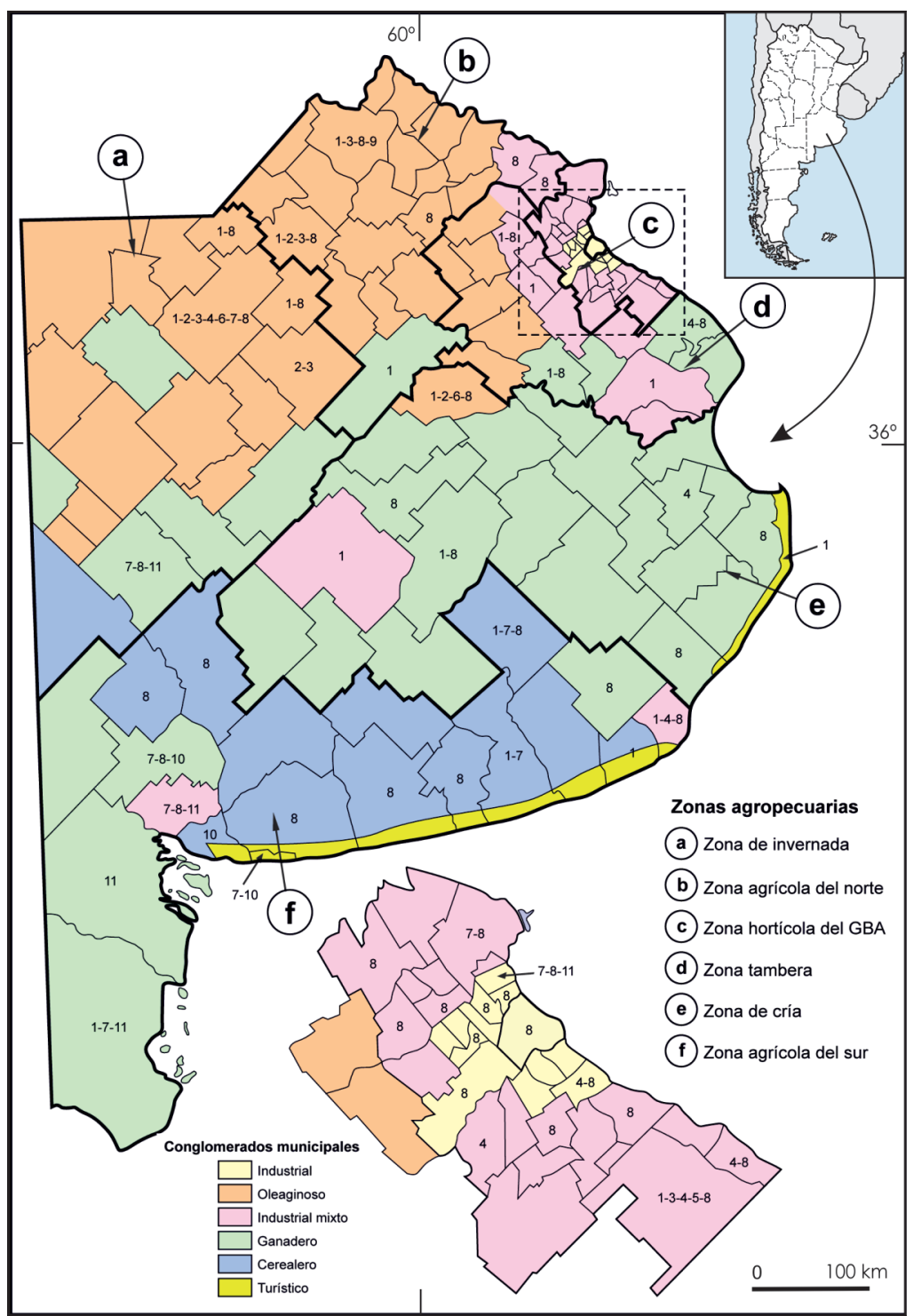

Fig. 1. Mapa de distribución de las hormigas del género Acromyrmex en la provincia de Buenos Aires. 1.A. ambiguus, 2. A. fracticornis, 3. A. heyeri, 4. A. hispidus, 5. A. hystrix, 6. A. landolti, 7. A. lobicornis, 8. A. lundii, 9. A. rugosus, 10. A. silvestrii, 11. A. striatus. Las líneas negras gruesas indican la separación de las zonas agropecuarias.

Las dos especies que se encuentran en todas las regiones geográficas son A. ambiguus y lundii, siguiendo en orden de importancia A. hispi$d u s$, presente en todas las regiones a excepción de la zona agrícola del norte, y en el otro extremo, A. rugosus que está presente únicamente en la mencionada zona. Con respecto a A. heyeri, siendo una especie graminícola, se encontró en la zona hortícola, agrícola del norte e invernada, no así en la tambera como de era de esperarse.

\section{DISCUSION}

Las localidades estudiadas por el equipo de investigación a lo largo de más de cuatro décadas representan casi el $62 \%$ de los datos reunidos en el presente trabajo: 54 de las 87 localidades relevadas, que corresponden a 56 partidos bonaerenses del total de 134 que conforman la provincia (Fig. 1). Los datos incluyen la corroboración de la distribución documentada de las especies: A. ambiguus, A. lobicornis y A. striatus para las localidades estudiadas. 

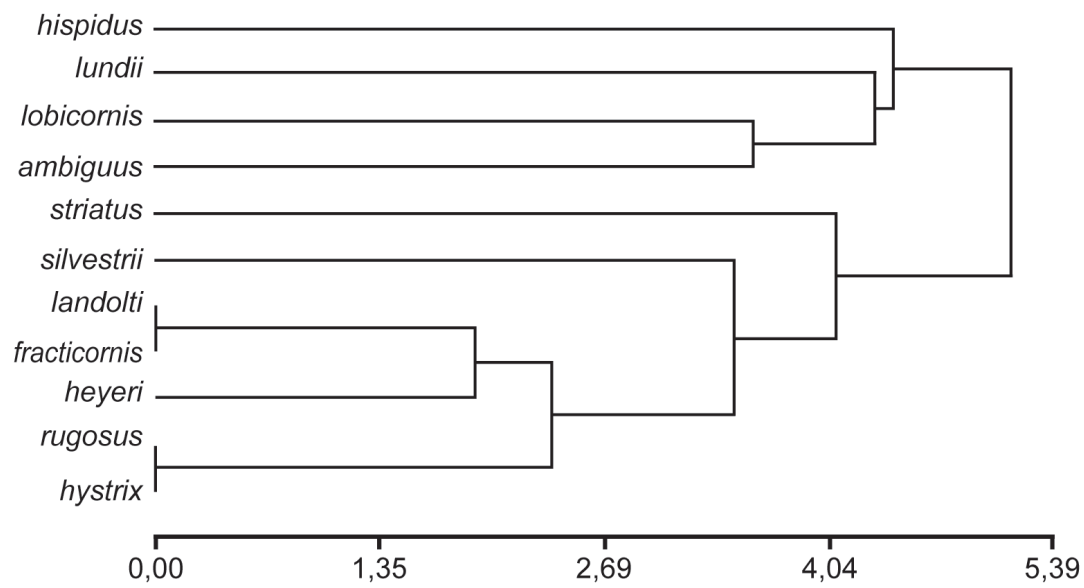

Fig. 2. Dendrograma de especies de Acromyrmex agrupadas de acuerdo a los coeficientes de distancia, en base a sus distribuciones en las distintas subregiones geográficas. Método WPGMA. Correlación cofenética= 0.873.

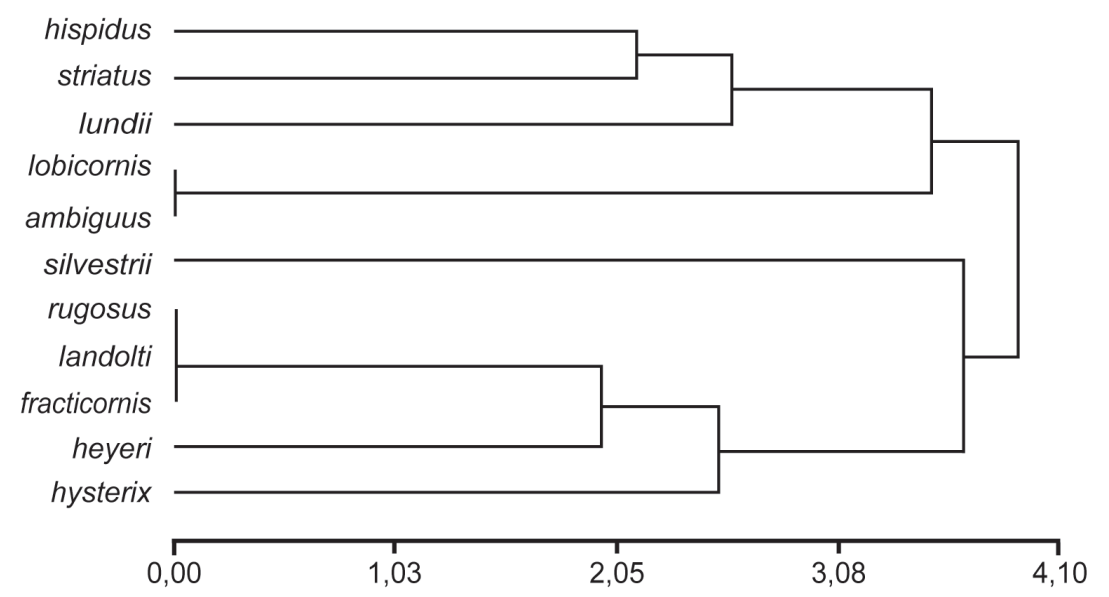

Fig. 3. Dendrograma de especies de Acromyrmex agrupadas de acuerdo a los coeficientes de distancia, en base a sus distribuciones en los conglomerados municipales. Método WPGMA. Correlación cofenética= 0.942 .

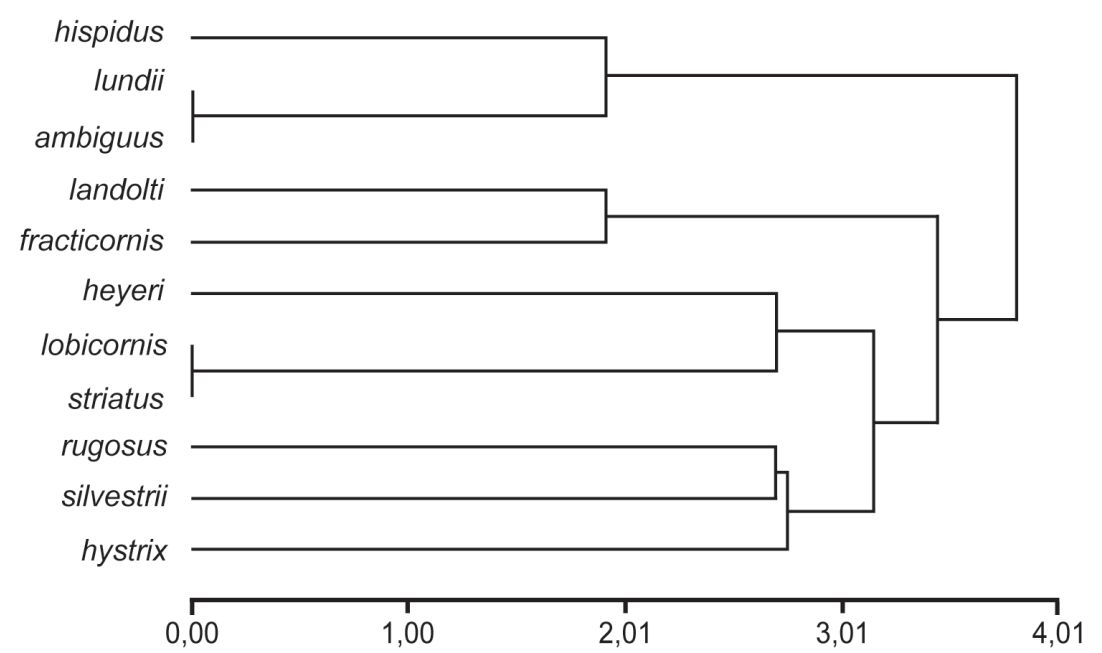

Fig. 4. Dendrograma de especies de Acromyrmex agrupadas de acuerdo a los coeficientes de distancias euclídeas, en base a sus distribuciones en zonas agropecuarias. Métodos WPGMA. Correlación cofenética= 0.935 . 
Como puede observarse en la Figura 1, A. lundii es la especie más ampliamente distribuida en todo el territorio provincial -43 partidos-, seguida por A. ambiguus -20 partidos- y A. lobicornis -10 partidos-. Al norte se encuentra A. rugosus -dos partido-, al nor-noroeste $A$. heyeri -cinco partidos-, centro-norte $A$. fracticornis -cuatro partidos-, centro-noroeste $A$. landolti -dos partidos-, al este $A$. hispidus -ocho partidos-, A. hystrix -un partido-, y al sur A. silvestri - tres partidos- y A. striatus -cinco partidos-.

Considerando los elevados coeficientes cofenéticos y los datos relevados a campo, el dendrograma que mejor explica la tendencia en la distribución de las especies de cortadoras sería el de las zonas agropecuarias (Fig. 4).

Cabe destacar que el único antecedente documentado sobre la distribución de la tribu Attini en Argentina menciona la presencia de cinco especies de Acromyrmex en la provincia de Buenos Aires sin diferenciar partidos ni localidades: $A$. lundii, lobicornis y striatus con amplia distribución en toda la provincia, $A$. heyeri solo en el norte y A. ambiguus en la Costa Atlántica. Nuestros estudios confirman la presencia de estas cinco especies, siendo A. ambiguus una especie muy frecuente, encontrándola además en el noroeste y centro de la provincia (Farji-Brener \& Ruggiero, 1994). Por otro lado los aportes mencionados sobre la distribución de A. heyeri en el norte bonaerense son coincidentes con nuestros hallazgos, a excepción de La Plata que se trata de un nuevo registro de localidad. Además, se da a conocer la presencia de cuatro especies: A. rugosus, A. hispidus, A. landolti y A. fracticornis para la provincia.

Las hormigas pueden encontrarse en todo tipo de hábitats desde el Círculo Polar Ártico hasta el Ecuador. El número de especies declina con la altitud, latitud y la aridez (Folgarait, 1998). Este hecho se comprueba aquí con respecto a la latitud, dado que de las 18 especies citadas para nuestro país sólo se registran 11 en territorio bonaerense.

Aunque el conocimiento de la fauna de hormigas en la provincia de Buenos Aires ha crecido considerablemente en los últimos 40 años, las contribuciones son fragmentarias. Se suma a este hecho el avance tanto de los monocultivos y como de las áreas industriales y urbanas en la región. El impacto que ello ocasiona impacta en la fauna edáfica entre las que se encuentran las hormigas cortadoras. La actividad industrial podría ocasionar la contaminación de los suelos, predominando así en ellos especies que mejor se adap- tan como se encontró en el presente estudio que únicamente $A$. hispidus, lundii y striatus están presentes en el conglomerado industrial (Fig. 3).

El género Acromyrmex se caracteriza por nidificar en el suelo, por lo cual las prácticas que realiza el hombre con fines productivos, industriales y urbanísticos afectan en forma directa su patrón de ocurrencia, predominando en cada una de las actividades aquellas especies mejor adaptadas. Esta situación deberá ser profundizada en futuros estudios que amplíen su conocimiento.

\section{AGRADECIMIENTOS}

A la Secretaría de Ciencia y Técnica de la Universidad Nacional de La Plata (UNLP) y a la Universidad Nacional del Noroeste de la provincia de Buenos Aires (UNNOBA) por su apoyo financiero; a dos revisores anónimos por sus comentarios constructivos sobre el manuscrito.

\section{BIBLIOGRAFÍA}

Agosti, D.; Majer; J. D. Alonso; L. E. \& Schultz, T. R. 2000. Standard Methods for Measuring and Monitoring Biodiversity. Smithsonian Institute Press, Washington DC.

Anglada, M.; Saluso, A.; Ermácora, O.; Maidana, A.; Dans, D. \& Decupyer, C. 2013. Hormigas podadoras: Estudios bioecológicos y alternativas de manejo en sistemas agrícolas y vegetación de monte en Entre Ríos. PID 2106. Ciencia, Docencia y Tecnología 3: 1-19.

AntWeb.2017:AntsofNeotropical.Disponibleen:https:// www.antweb.org/bioregion.do? name $=$ Neotropical. Acceso 28 de Septiembre de 2016.

Bestelmeyer, B.T. \& Wiens J. A. 1996. The effects of land use on the structure of ground-foraging ant communities in the Argentine chaco. Ecological Applications 6: 1225-1240.

Blondel, D.; Sgarbi, C.; Culebra Mason, S. \& Ricci, M. 2010. Evaluación de atrayentes alimentarios para la captura de hormigas cortadoras (Formicidae: Attini) en sistemas productivos de la localidad de Lincoln. Seminario-Taller "Hormigas cortadoras de hoja asociadas a los sistemas agroforestales" Facultad de Ciencias Agropecuarias. UNER-Oro Verde.

Blondel, D.; Sgarbi, C.; Verzero Villalba, F; Catalano, P.; Culebra Mason, S. \& Ricci, M. 2009. Hormigas cortadoras (Hymenoptera: Formicidae): utilización de atrayentes alimentarios para su implementación en planes de monitoreo. XIII Jornadas Fitosanitarias Argentinas. Termas de Rio Hondo, Santiago del Estero, Argentina.

Bolton, B. 2016. An Online Catalogue of the Ants of the World Disponible en http://antcat.org (acceso septiembre 2016).

Brown, B.V.; Henry L. Disney; R.; Elizalde, L. \& 
Folgarait, P.J. 2010. New Species and New Records of Apocephalus Coquillett (Diptera: Phoridae) that Parasitize Ants (Hymenoptera: Formicidae) in America. Sociobiology 55 (1): 165-190.

Bruch, C. 1916. Contribución al estudio de las hormigas de San Luis. Revista del Museo de La Plata 23: 291-357.

Bruch, C. 1917. Costumbres y nidos de hormigas. Anales de la Sociedad Científica Argentina, Buenos Aires 83: 302-316.

Bruch, C. 1918. Comunicaciones entomológicas III. Huéspedes de la hormiga negra recolectados por el Sr. Juan Bosq. Physis 4: 358-359.

Bruch, C. 1921. Estudios mirmecológicos. Revista del Museo de La Plata 26: 175-211.

Calcaterra, L. A.; Briano, J. A. \& Williams D. F. 2000. New Host for the Parasitic Ant Solenopsis daguerrei (Hymenoptera: Formicidae) in Argentina. The Florida Entomologist 83 (3): 363-365.

Calcaterra, L.; Cabrera, S. \& Briano, J. 2016. Local cooccurrence of several highly invasive ants in their native range: are they all ecologically dominant species? Insectes Sociaux 63:407-419

Cappello, V. \& Fortunato, N. 2008. Plaguicidas en la provincia de Buenos Aires: información toxicológica, ecotoxicológica y aspectos ambientales. Dirección Provincial de Recursos Naturales, Programa de Gestión Ambiental. 138 páginas.

Catalano, P.; Culebra Mason, M.; Ricci, M; Sgarbi, C.; Verzero Villalba, F. \& Blondel, D. 2008. Utilización de trampas Pitfall con distintos atrayentes alimentarios para el monitoreo de hormigas en sistemas pastoriles. VII Congreso Argentino Entomología. Huerta Grande Córdoba.

Catalano, P.; Culebra Mason, M.; Sgarbi, C. \& Ricci, M. 2012. Primeros aportes al conocimiento de la Mirmecofauna presente en un establecimiento agrícola-ganadero de la localidad de Saladillo. Revista de la Facultad de Agronomía (La Plata) 111(1): 31-40.

Cherrett, J. M.; Powell, R. J. \& Stradling, D. J. 1989. The mutualism between leaf-cutting ants and their fungus. En: Wilding, N., N.M. Colling \& J.F. Webber (eds.), Insect Fungus Interactions, pp. 93120. Academic Press, London.

Cuezzo, F. 1998. Formicidae. Pp. 452-462. En: Morrone, J. J.; Coscarón, S. (eds.) 1998. Biodiversidad de artrópodos argentinos. La Plata: Ediciones Sur, vii + $599 \mathrm{pp}$.

Culebra Mason, M. S.; Sgarbi, C.; Catalano, P.; Blondel, D.; Verzero Villalba, F. \& Ricci, M. 2008. Diversidad de mirmecofauna presente en dos agroecosistemas de la provincia de Bs. As. VII Congreso Argentino de Entomología. Huerta Grande, Córdoba.

Culebra Mason, M. S.; Sgarbi, C.; Catalano, P.; Blondel, D.; Verzero Villalba, F. \& Ricci, M. 2009. Identificación de especies de Formicidos presentes en un cultivo de soja. XIII Jornadas Fitosanitarias Argentinas. Termas de Rio Hondo, Santiago del Estero.

De Coll, O. inéd. Identificación, bioecología y forrajeo de hormigas cortadoras de los géneros Atta y
Acromyrmex (Hymenoptera: Formicidae) en ambientes agropecuarios y forestales en Misiones, Argentina. Tesis para optar al Título de Doctor en Biología, Facultad de Ciencias Exactas y Naturales y Agrimensura, Universidad Nacional de Nordeste, Corrientes, Argentina.

De Santis, L. 1941. II. Las hormigas podadoras. En: Las principales hormigas dañinas de la Provincia de Buenos Aires. Dirección de Agricultura, Ganadería e Industrias, Ministerio de Obras Públicas de la Provincia de Buenos Aires, La Plata, Pasaje Dardo Roca: 1-40.

Di Rienzo, J.A.; Casanoves, F.; Balzarini, M.G.; Gonzalez, L.; Tablada, M. \& Robledo, C.W. 2008. InfoStat, versión 2008. Grupo InfoStat, FCA, Universidad Nacional de Córdoba, Argentina.

De Souza, D. J.; Fernándes Soares, I. M. \& Castro Della Lucia, T. M. 2007. Acromyrmex ameliae sp. $n$. (Hymenoptera: Formicidae): A new social parasite of leaf-cutting ants in Brazil. Insect Science 14 (3): 251-257.

Di Iorio, O. \& Turienzo, P. 2015. Campo de Mayo: un área natural histórica en los alrededores de la ciudad de Buenos Aires, Argentina, que merece ser declarada reserva natural. Revista del Museo Argentino de Ciencias Naturales 17(1): 13-42.

EcoRegistros. 2011-2015. Registros Ecológicos de la Comunidad. Ecoregistros.org http://www.ecoregistros.org.

Falibene, A. F. 2012. Modulación del comportamiento alimentario en hormigas: fisiología de la ingestión de néctar. Tesis presentada para optar al título de Doctor de la Universidad de Buenos Aires en el área Ciencias Biológicas. 147 páginas.

Farji-Brener, A. G. \& Ruggiero, A. 1994. Leaf-cutting ants (Atta and Acromyrmex) inhabiting Argentina: Patterns in species richness and geographical range sizes. Journal of Biogeography 21: 391-399.

Fernández, F. \& Sendoya, S. 2004. List of Neotropical Ants Genera. Biota Colombiana 5: 3-93.

Fernandez Valiela, M.S.; Bakarcic, M. \& Turica, A. 1954. Manual de enfermedades y plagas de los frutales y forestales en el Delta del Paraná. Dirección General de Investigación Agrícola. Centro Regional Pampeano (Sección Agrícola). Laboratorio de Fitopatología del Delta del Paraná. Ministerio de Agricultura y Ganadería, Bs. As., Publicación miscelánea № 400. 1-192.

Fisher, B. \& Fong, J. 2017. AntWeb. California Academy of Sciences. Occurrence Dataset https:// doi.org/10.15468/wqmjjt_(último acceso junio 2017 vía GBIF.org).

Folgarait, P. 1998. Ant biodiversity and its relationship to ecosystem functioning: a review. Biodiversity and conservation 7: 1221-1244.

Galarza, J. G. 1985. Ensayo de control químico de hormigas cortadoras de hojas y hormigas de fuego. Acintacnia 16: 38-40.

Gallardo, A. 1915. Observaciones sobre algunas hormigas de la R. A. Anales del Museo Nacional de Historia, Buenos Aires 27 (111): 1-35.

Gallardo, A. 1916 a. Fauna mirmecológica de Tandil y 
Ventana. Physis 2(10): 128-131.

Gallardo, A. 1916 b. Notes sistematiques et ethologiques sur les fourmis "Attines" de la Republique Argentine. Anales del Museo Nacional de Buenos Aires 28 (124): 317-344.

Gonçalves, C.R. 1961. O Genero Acromyrmex no Brasil (Hym. Formicidae). Studia Entomologica 4(1-4): 113-174.

Guido, S. 2011. Control de hormigas cortadoras. Visión Rural V 18 (89); 48-49.

Harada, A. 2016. State of Art of Ants (Hymenoptera: Formicidae) at Caxiuanã, Melgaco, Pará, Brazil. Advances in Entomology 4: 115-132. doi: 10.4236/ ae.2016.43013.

Hölldobler, B. \& Wilson, E.O. 1990. The Ants. Harvard University Press, Cambridge. http://dx.doi. org/10.1007/978-3-662-10306-7.

Hölldobler, B. \& Wilson, E. O. 2011. The leafcutter ants. W.W. Norton, New York, London, 160 páginas.

Janicki, J.; Narula, N.; Ziegler, M.; Guénard \& Economo, E.P. 2016. Visualizing and interacting with largevolume biodiversity data using client-server webmapping applications: The design and implementation of antmaps.org. Ecological Informatics 32: 185-193. http://antmaps.org/about.html (último acceso agosto 2017).

Johnson, S.C. 1967. Hierarchical clustering schemes. Psychometrika 32: 241-254.

Kusnezov, N. 1978. Hormigas argentinas. Clave para su identificación. Edición preparada por R. Golbach, Fundación Miguel Lillo, Miscelánea 61: 35-139.

Lazzarini, A.; Brescia, V. \& Rivera, I. 2004. Avances en el análisis del CNA 2002 y su comparación con el CNA 1988. Documento de difusión, INTA, Instituto de Economía y Sociología.

Loiácono, M. S.; Margaría, C. \& Aquino, D. A. 2013. Diapriinae wasps (Hymenoptera: Diaprioidea: Diapriidae) associated with ants (Hymenoptera: Formicidae) in Argentina. Psyche, vol. 2013, Article ID 320590, 11 pages, doi:10.1155/2013/320590.

Lugo, M.; Crespo, A.; Esteban, M; Cafaro, M. \& Jofre, L. 2013. Hongos asociados con dos poblaciones de Acromyrmex lobicornis (Formicidae) de San Luis, Argentina. Boletín de la Sociedad Argentina de Botánica 48 (1): 5-15.

Mac Donagh, E. J. 1937. Hormigas podadoras del extremo Sur de Bs. As. Notas del Museo de La Plata 2 (Zool. 5): 45-52.

Mac Donagh E.J. 1939. Las hormigas "Attinae" de Patagones y rectificación de la supuesta "Oecodoma" de Hudson. Physis Buenos Aires 17: 211-215.

Mc Quitty, L.L. 1966. Similarity analysis by reciprocal pairs for discrete and continuous data. Educational and Psychological Measurements 26: 825-831.

Majer, J. 1983. Ants: bioindicators of mine-site rehabilitation, land use and land conservation. Enviromental Management 7(4): 375-383.

Martinez, C.; De Haro, A. M.; Barañao, J.; Riquelme Virgala, M. B. \& Santadino, M. 2005. Herbivoría de Acromyrmex lundii (Hymenoptera: Formicidae) según orígenes de Eucalyptus spp. Revista de la
Sociedad Entomológica Argentina 64(4) 286-287.

Montalti, D.; Ferman, L. M.; Camperi, A. R.; Soave, G. E.; Arambarri, A. M. \& Darrieu, C. A. 2005. Winter Diet of Great Pampa-Finches Embernagra platensis in Guaminí Lagoon, Argentina. Ornithologica 40 (1): 79-82.

Morello, J.; Buzai, J. D.; Baxendale, C. A.; Rodríguez, A. F.; Matteucci, S. D.; Godagnone, R. E. \& Casas R. R. 2000. Urbanization and the consumption of fertile land and other ecological changes: the case of Buenos Aires. Environment and Urbanization 12 (2): 119-131.

Morrone, J. J. 2001. Biogeografía de América Latina y el Caribe. M\&T-Manuales \& Tesis SEA, vol. 3. Zaragoza, $148 \mathrm{pp}$.

Nobua Behrmann, B.E. 2014. Interacciones tróficas entre dos especies simpátricas de hormigas cortadoras y el ensamble de plantas en el Monte central. Facultad de Ciencias Exactas y Naturales. Universidad de Buenos Aires URL: http://digital.bl.fcen.uba.ar/Download/ Tesis/Tesis_5456_NobuaBehrmann.pdf http://digital.bl.fcen.uba.ar/gsdl-282/cgi-bin/ library.cgi $? \mathrm{a}=\mathrm{d} \& \mathrm{c}=$ tesis $\& \mathrm{~d}=$ Tesis_5456_ NobuaBehrmann

Peralta, L. \& Martinez, P. A. 2013. Ensambles de ácaros oribátidos en hormigueros de Acromyrmex spp. (Hymenoptera, Formicidae). Ecología austral 23 (3): 209-217.

Pompozzi, G.A. inéd. Estudio de la diversidad de arañas (Araneae) en cultivos invernales de la provincia de Buenos Aires y su importancia como enemigos naturales de insectos plaga. Tesis Doctoral, Universidad Nacional del Sur. Departamento de Biología, Bioquímica y Farmacia. Bahía Blanca.

Quesada Aramburú, J. \& Cadelli, E. 2012. Hacia una clasificación de los municipios bonaerenses. Documento de Trabajo DPEPE N04/2012.

Ricci, M.; Benítez, D.; Padín, S. \& Maceiras, A. 2005. Hormigas Argentinas: Comportamiento, Distribución y Control. http://www.produccion-animal.com.ar/produccion_y_manejo_pasturas/pasturas_combate_de_plagas_y_malezas/49-hormigas_ argentinas.pdf (último acceso diciembre 2016)

Ricci, M.; Culebra Mason, S.; Sgarbi, C.; Peña, J.; Catalano, P.; Verzero Villalba, F.; Blondel; D. \& Faropa, D. 2012. Aportes al conocimiento de la diversidad de hormigas (Hymenoptera: Formicidae) en cinco localidades de la Provincia. de Bs. As. VIII Congreso Argentino de Entomología, Bariloche.

Ricci, M.; Sgarbi, C.; Culebra Mason, S.; Peña, J. \& Chila Covachina, J. 2015. Diversidad de especies de hormigas cortadoras del género Acromyrmex (Hymenoptera, Formicidae) en el noroeste de la provincia de Buenos Aires, Argentina. IX Congreso Argentino de Entomología, Posadas.

SAGyP. 1995. El deterioro de las tierras en la República Argentina: alerta amarilla. Buenos Aires: Argentina. Secretaría de Agricultura Ganadería, Pesca y Alimentos. Subsecretaría de Alimentación; Consejo Federal Agropecuario en Alerta Amarillo; 286 páginas. 
Santschi, F. 1916. Formicides sudaméricains, nouveaux ou peu connus. Physis Buenos Aires 2: 365-399.

Slutzky, D. 1968. Aspectos sociales del desarrollo rural en la pampa húmeda argentina. Desarrollo económico 29 (8): 95-136.

Sokal, R.R. \& Michener, C.D. 1958. A Statistical Methods for Evaluating Systematic Relationships. University of Kansas Science Bulletin 38: 14091438.

Tizón, R.; Wulff, J.P. \& Peláez, D.V. 2014. The effect of increase in the temperature on the foraging of Acromyrmexlobicornis (Hymenoptera: Formicidae). Zoological Studies 53: 40. doi:10.1186/s40555-0140040-4.

Verzero Villalba, F.; Sgarbi, C.; Culebra Mason, S. \& Ricci, M. 2011. Grupos funcionales de hormigas: evaluación de su utilización como indicadores del impacto ambiental producto de la agricultura y el cambio climático. Documento Preliminar: Parte D. III Congreso Internacional de Cambio Climático y desarrollo sustentable. Ecosistemas (54). Anexo. Habitat. Global Argenta. https://es.scribd.com/ document/66680258/Documento-PreliminarParte-D-3er-Congreso-de-Cambio-Climatico-yDesarrollo-Sustentable.

Verzero Villalba, F.; Sgarbi, C.; Culebra Mason, S. \& Ricci, M. 2014. Grupos funcionales dominantes de hormigas (Hymenoptera: Formicidae) en pastizales naturales con y sin pastoreo del noroeste de Buenos Aires, Argentina. Revista de la Facultad de Agronomía, La Plata 113 (2): 107-113.

Vittar, F. \& Cuezzo, F. C. 2008. Hormigas (Hymenoptera: Formicidae) de la provincia de Santa Fe, Argentina. Revista de la Sociedad Entomológica Argentina 67(1-2): 175-178.

Ward P. S.; Brady, S. G.; Fisher, B. L. \& Schultz, T. R. 2015. The evolution of myrmicine ants: phylogeny and biogeography of a hyperdiverse ant clade (Hymenoptera: Formicidae). Systematic Entomology 40: 61-81.

Doi: 10.22179/REVMACN.19.483

Recibido: 16-I-2017

Aceptado: 9-XI-2017 


\section{Apéndice}

Especies de hormigas del género Acromyrmex Mayr. Distribución geográfica (partido y localidad), referencia bibliográfica, ubicación en los conglomerados municipales (C), zonas agropecuarias (ZA) (Aramburu \& Cadelli, 2012), y subregiones (SR) (SAGyP, 1995). C= I: Industrial, IM: Industrial Mixto, O: Oleaginoso, G: Ganadero, C: Cerealero, T: Turístico. ZA= a: Hortícola, b: Tambera, c: Agrícola del Norte, d: de Invernadas, e: de cría, f: Agrícola del Sur. SR= TV: Tandilia y Ventania, PO: Pampa Ondulada, PI: Pampa Interserrana, PD: Pampa Deprimida, PA: Pampa Arenosa, SP: Sector Patagónico, M: Metropolitana, D: Delta. RL: Relacionada a lagos. *nuevo registro de localidad, **corroboración de distribución.

\begin{tabular}{|c|c|c|c|c|}
\hline \multicolumn{5}{|c|}{ 1. Acromyrmex ambiguus } \\
\hline Partido: Localidad & Referencia bibliográfica & $\mathrm{C}$ & $\mathrm{ZA}$ & SR \\
\hline Azul: Azul & * & G & $\mathrm{e}$ & TV \\
\hline Chascomús: Chascomús & * & IM & $\mathrm{b}$ & $\mathrm{PO}$ \\
\hline Gral. Alvarado: Miramar & Gallardo, 1916 b** & $\mathrm{C}$ & $\mathrm{d}$ & PI \\
\hline Gral. Las Heras: Gral. Las Heras & Gallardo, 1916 b, Mac Donagh, 1937, 1939 & $\mathrm{O}$ & $\mathrm{b}$ & $\mathrm{PO}$ \\
\hline $\begin{array}{l}\text { Gral. Pueyrredón: Reserva Laguna } \\
\text { de los Padres }\end{array}$ & Peralta \& Martínez, 2013 & IM & $\mathrm{f}$ & TV \\
\hline Gral. Viamonte: Gral. Viamonte & * & $\mathrm{O}$ & $\mathrm{d}$ & $\mathrm{PA}$ \\
\hline Junín: Junín & Ricci et. al, 2012, 2015 & $\mathrm{O}$ & $\mathrm{c}$ & $\mathrm{PA}$ \\
\hline La Costa: San Bernardo del Tuyú & * & $\mathrm{T}$ & $\mathrm{e}$ & $\mathrm{PD}$ \\
\hline La Plata: La Plata & $*$ & IM & $\mathrm{a}$ & $\mathrm{PO}$ \\
\hline La Plata: Gonnet & * & IM & $\mathrm{a}$ & $\mathrm{PO}$ \\
\hline Leandro N. Alem: Vedia & * & $\mathrm{O}$ & $\mathrm{d}$ & $\mathrm{PA}$ \\
\hline Lincoln: Arenaza & Blondel et al., 2010 & $\mathrm{O}$ & $\mathrm{d}$ & $\mathrm{PA}$ \\
\hline Lincoln: Lincoln & Blondel et al., 2009, Ricci et al., 2012, 2015 & $\mathrm{O}$ & $\mathrm{d}$ & $\mathrm{PA}$ \\
\hline Luján: San Eladio & Calcaterra et al., 2000 & IM & $\mathrm{b}$ & $\mathrm{PO}$ \\
\hline Monte: San Miguel del Monte & * & $\mathrm{G}$ & $\mathrm{b}$ & $\mathrm{PO}$ \\
\hline Necochea: Necochea & * & $\mathrm{C}$ & $\mathrm{d}$ & PI \\
\hline Necochea: Quequén & * & $\mathrm{C}$ & $\mathrm{f}$ & PI \\
\hline Olavarría: Olavarría & * & IM & $\mathrm{e}$ & TV \\
\hline Patagones: Carmen de Patagones & Mac Donagh, 1937, 1939 & G & $\mathrm{f}$ & SP \\
\hline Pergamino: Pergamino & $*$ & $\mathrm{O}$ & $\mathrm{c}$ & $\mathrm{PO}$ \\
\hline Saladillo: Saladillo & $\begin{array}{l}\text { Catalano et al., 2008, 2012; Culebra Mason et al., } \\
\text { 2008, 2009; Ricci et al., 2012, } 2015\end{array}$ & $\mathrm{O}$ & e & PA \\
\hline Tandil: Tandil & * & $\mathrm{C}$ & $\mathrm{f}$ & TV \\
\hline Veinticinco de Mayo: Riestra & * & G & $\mathrm{c}$ & PA \\
\hline
\end{tabular}

\begin{tabular}{|l|l|c|c|c|}
\hline \multicolumn{1}{|c|}{ 2. Acromyrmex fracticornis } \\
\hline \multicolumn{1}{|c|}{ Partido: Localidad } & \multicolumn{1}{|c|}{ Referencia bibliográfica } & $\mathrm{C}$ & ZA & SR \\
\hline Junín: Junín & $*$ & $\mathrm{O}$ & $\mathrm{c}$ & PA \\
\hline Lincoln: Lincoln & $*$ & $\mathrm{O}$ & $\mathrm{d}$ & PA \\
\hline 9 de Julio: Carlos María Naón & Verzero Villalba et al., 2011; Ricci et al., 2012 & $\mathrm{O}$ & $\mathrm{d}$ & PA \\
\hline Saladillo: Saladillo & $\begin{array}{l}\text { Culebra Mason } \text { et al., 2008, 2009; Ricci } \text { et al., 2012, } \\
2015\end{array}$ & $\mathrm{O}$ & $\mathrm{e}$ & $\mathrm{PA}$ \\
\hline
\end{tabular}

\begin{tabular}{|l|l|c|c|c|}
\hline \multicolumn{1}{|c|}{ 3. Acromyrmex heyeri } \\
\hline \multicolumn{1}{|c|}{ Partido: Localidad } & \multicolumn{1}{|c|}{ Referencia bibliográfica } & C & ZA & SR \\
\hline Junín: Junín & Ricci et al., 2012, 2015 & O & c & PA \\
\hline La Plata: Isla Martín García & $\begin{array}{l}\text { Santschi, 1916; Kusnezov, 1978; Bruch, 1921; De } \\
\text { Santis, 1941 }\end{array}$ & IM & a & PO \\
\hline La Plata: La Plata & $*$ & IM & a & PO \\
\hline Lincoln: El Triunfo & $*$ & O & d & PA \\
\hline
\end{tabular}




\begin{tabular}{|c|c|c|c|c|}
\hline \multicolumn{5}{|c|}{ 3. Acromyrmex heyeri } \\
\hline Partido: Localidad & Referencia bibliográfica & $\mathrm{C}$ & ZA & SR \\
\hline 9 de Julio: Carlos María Naón & Verzero Villalba et al., 2011 Ricci et al., 2012, 2015 & $\mathrm{O}$ & $\mathrm{d}$ & $\mathrm{PA}$ \\
\hline Pergamino: Pergamino & Ricci et. al., 2012, 2015 & $\mathrm{O}$ & $\mathrm{c}$ & $\mathrm{PO}$ \\
\hline
\end{tabular}

\begin{tabular}{|c|c|c|c|c|}
\hline \multicolumn{5}{|c|}{ 4. Acromyrmex hispidus } \\
\hline Partido: Localidad & Referencia bibliográfica & $\mathrm{C}$ & $\mathrm{ZA}$ & SR \\
\hline Berisso: Berisso & * & $\mathrm{I}$ & $\mathrm{a}$ & $\mathrm{PO}$ \\
\hline Dolores: Dolores & * & $\mathrm{G}$ & $\mathrm{e}$ & $\mathrm{PD}$ \\
\hline Ezeiza: Ezeiza & * & $\mathrm{IM}$ & $\mathrm{a}$ & $\mathrm{M}$ \\
\hline $\begin{array}{l}\text { Gral. Pueyrredón: Sierra de los } \\
\text { Padres* }\end{array}$ & * & $\mathrm{IM}$ & $\mathrm{f}$ & TV \\
\hline La Plata: Villa Castells & * & IM & $\mathrm{a}$ & $\mathrm{PO}$ \\
\hline Lincoln: Arenaza & Blondel et al., 2010; Ricci et al., 2012, 2015 & $\mathrm{O}$ & $\mathrm{d}$ & $\mathrm{PA}$ \\
\hline Magdalena: Gral. Mansilla & * & $\mathrm{G}$ & $\mathrm{b}$ & $\mathrm{PO}$ \\
\hline Magdalena: Arditi & * & $\mathrm{G}$ & $\mathrm{b}$ & $\mathrm{PO}$ \\
\hline Quilmes: Quilmes & * & $\mathrm{I}$ & $\mathrm{a}$ & $\mathrm{M}$ \\
\hline
\end{tabular}

\begin{tabular}{|c|c|c|c|c|}
\hline \multicolumn{1}{|c|}{ 5. Acromyrmex hystrix } & C & ZA & SR \\
\hline Partido: Localidad & Referencia bibliográfica & IM & a & PO \\
\hline La Plata: La Plata & Fisher \& Fong, 2017 & .
\end{tabular}

\begin{tabular}{|c|c|c|c|c|}
\hline \multicolumn{5}{|c|}{ 6. Acromyrmex landolti } \\
\hline Partido: Localidad & Referencia bibliográfica & $\mathrm{C}$ & $\mathrm{ZA}$ & SR \\
\hline Lincoln: Lincoln & Ricci et al., 2012, 2015 & $\mathrm{O}$ & $\mathrm{d}$ & $\mathrm{PA}$ \\
\hline Saladillo: Cazón & Catalano et al., 2012 Ricci et al., 2012, 2015 & $\mathrm{O}$ & $\mathrm{e}$ & $\mathrm{PA}$ \\
\hline
\end{tabular}

\begin{tabular}{|c|c|c|c|c|}
\hline \multicolumn{5}{|c|}{ 7. Acromyrxmex lobicornis } \\
\hline Partido: Localidad & Referencia bibliográfica & $\mathrm{C}$ & ZA & SR \\
\hline Bahía Blanca: Bahía Blanca & Tizón et al., 2014 & IM & $\mathrm{f}$ & $\mathrm{SP}$ \\
\hline Guaminí: Laguna Guaminí & Montalti et al., 2005 & $\mathrm{G}$ & $\mathrm{d}$ & $\mathrm{RL}$ \\
\hline Lincoln: Arenaza & * & $\mathrm{O}$ & $\mathrm{d}$ & $\mathrm{PA}$ \\
\hline Monte Hermoso: Monte Hermoso & EcoRegistros & $\mathrm{T}$ & $\mathrm{f}$ & TV \\
\hline Necochea: Quequén & * & $\mathrm{C}$ & $\mathrm{f}$ & $\mathrm{PI}$ \\
\hline Patagones: Carmen de Patagones & Mac Donagh, 1937, 1939** & $\mathrm{G}$ & $\mathrm{f}$ & $\mathrm{SP}$ \\
\hline San Isidro: San Isidro & * & $\mathrm{I}$ & $\mathrm{a}$ & $\mathrm{PO}$ \\
\hline Tandil: Tandil & Gallardo, 1915; 1916 a y b; De Santis, 1941 & $\mathrm{C}$ & $\mathrm{f}$ & TV \\
\hline Tigre: Delta & Fernandez Valiela et al., 1954 & IM & a & $\mathrm{D}$ \\
\hline Tornquist: Sierra de la Ventana & De Santis, 1941; Bruch, 1917, 1921 & $\mathrm{G}$ & $\mathrm{f}$ & TV \\
\hline
\end{tabular}

\begin{tabular}{|l|l|c|c|c|}
\hline \multicolumn{1}{|c|}{ 8. Acromyrmex lundii } & \multicolumn{1}{c|}{ Referencia bibliográfica } & C & ZA & SR \\
\hline Almirante Brown: Longchamps & EcoRegistros & IM & a & M \\
\hline Azul: Chillar & $*$ & G & e & TV \\
\hline Bahía Blanca: Bahía Blanca & $*$ & IM & f & SP \\
\hline Balcarce: Balcarce & Guido, 2011 & G & f & TV \\
\hline Berazategui: Berazategui & $*$ & IM & a & M \\
\hline Berazategui: Hudson & EcoRegistros & IM & a & M \\
\hline Berisso: Berisso & $*$ & IM & a & PO \\
\hline $\begin{array}{l}\text { Ciudad Autónoma Buenos Aires } \\
\text { (CABA): } \text { Flores }\end{array}$ & Bruch, 1918 & I & a & M \\
\hline
\end{tabular}




\begin{tabular}{|c|c|c|c|c|}
\hline \multicolumn{5}{|c|}{ 8. Acromyrmex lundii } \\
\hline Partido: Localidad & Referencia bibliográfica & $\mathrm{C}$ & ZA & SR \\
\hline $\begin{array}{l}\text { CABA: Reserva ecológica Ciudad } \\
\text { Universitaria - Costanera Norte }\end{array}$ & EcoRegistros & I & a & $\mathrm{M}$ \\
\hline CABA: Mataderos & * & I & $\mathrm{a}$ & $\mathrm{M}$ \\
\hline $\begin{array}{l}\text { CABA: Sede de Ciudad Universitaria } \\
\text { Costanera (UBA) }\end{array}$ & Falibene, 2012 & $\mathrm{I}$ & a & $\mathrm{M}$ \\
\hline $\begin{array}{l}\text { Campana: Reserva Natural de } \\
\text { Otamendi }\end{array}$ & Calcaterra et al., 2016 & $\mathrm{IM}$ & $\mathrm{c}$ & $\mathrm{D}$ \\
\hline Carmen de Areco: Carmen de Areco & Brown et al., 2010 & $\mathrm{O}$ & $\mathrm{c}$ & $\mathrm{PO}$ \\
\hline Cnel. Dorrego: Cnel. Dorrego & * & $\mathrm{C}$ & $\mathrm{f}$ & TV \\
\hline Cnel. Suárez: Cnel. Suárez & ** & $\mathrm{C}$ & $\mathrm{f}$ & TV \\
\hline Del Pilar: Reserva Natural del Pilar & EcoRegistros & IM & $\mathrm{a}$ & $\mathrm{PO}$ \\
\hline Gral. Lavalle: Gral. Lavalle & * & $\mathrm{G}$ & $\mathrm{e}$ & $\mathrm{PD}$ \\
\hline Gral. Pueyrredón: Mar del Plata & * & IM & $\mathrm{f}$ & TV \\
\hline Gral. Pueyrredón: Chapalmalal & * & IM & $\mathrm{f}$ & TV \\
\hline $\begin{array}{l}\text { Gral. Pueyrredón: Reserva Laguna de } \\
\text { los Padres }\end{array}$ & Peralta \& Martínez, 2013 & IM & $\mathrm{f}$ & TV \\
\hline Gral. San Martín: San Martín & * & $\mathrm{I}$ & $\mathrm{a}$ & $\mathrm{M}$ \\
\hline Gral. Viamonte: Gral. Viamonte & * & $\mathrm{O}$ & $\mathrm{d}$ & $\mathrm{PA}$ \\
\hline Guaminí: Laguna Guaminí & Montalti et al., 2005 & G & $\mathrm{d}$ & $\mathrm{RL}$ \\
\hline Junin: Junín & Ricci et al., 2012, 2015 & $\mathrm{O}$ & $\mathrm{c}$ & $\mathrm{PA}$ \\
\hline La Matanza: Ramos Mejía & EcoRegistros & $\mathrm{I}$ & $\mathrm{a}$ & $\mathrm{M}$ \\
\hline La Matanza: San Justo & EcoRegistros & $\mathrm{I}$ & $\mathrm{a}$ & $\mathrm{M}$ \\
\hline La Plata: Arana & * & IM & $\mathrm{a}$ & $\mathrm{PO}$ \\
\hline La Plata: Isla Martín García & * & IM & $\mathrm{a}$ & $\mathrm{PO}$ \\
\hline La Plata: Los Hornos & * & IM & $\mathrm{a}$ & $\mathrm{PO}$ \\
\hline La Plata: La Plata & Loiácono et al., 2013 & IM & $\mathrm{a}$ & $\mathrm{PO}$ \\
\hline $\begin{array}{l}\text { La Plata: Parque Provincial Pereyra } \\
\text { Iraola }\end{array}$ & * & IM & a & $\mathrm{PO}$ \\
\hline La Plata: Villa Elisa & * & IM & $\mathrm{a}$ & $\mathrm{PO}$ \\
\hline La Plata: Tolosa & * & IM & $\mathrm{a}$ & $\mathrm{PO}$ \\
\hline Leandro N. Alem: Vedia & * & $\mathrm{O}$ & $\mathrm{d}$ & $\mathrm{PA}$ \\
\hline Lincoln: Arenaza & $\begin{array}{l}\text { Blondel et al., 2009, 2010; Culebra Mason et al., } \\
2008\end{array}$ & $\mathrm{O}$ & d & $\mathrm{PA}$ \\
\hline Lincoln: Lincoln & Ricci et al., 2012, 2015 & $\mathrm{O}$ & $d$ & $\mathrm{PA}$ \\
\hline Luján: Luján & Martinez et al., 2005 & IM & $\mathrm{b}$ & $\mathrm{PO}$ \\
\hline Luján: San Eladio & Calcaterra et al., 2000 & IM & $\mathrm{b}$ & $\mathrm{PO}$ \\
\hline Magdalena: Gral. Mansilla & * & G & $\mathrm{b}$ & $\mathrm{PO}$ \\
\hline Magdalena: Vieytes & * & G & $\mathrm{b}$ & $\mathrm{PO}$ \\
\hline $\begin{array}{l}\text { Mar Chiquita: Albúfera de Mar } \\
\text { Chiquita }\end{array}$ & EcoRegistros & G & $\mathrm{e}$ & PD \\
\hline Monte: San Miguel del Monte & EcoRegistros & $\mathrm{G}$ & $\mathrm{b}$ & $\mathrm{PO}$ \\
\hline Moreno: Moreno & Brown et al., 2010 & IM & $\mathrm{a}$ & $\mathrm{M}$ \\
\hline Morón: Castelar & Brown et al., 2010; EcoRegistros; Galarza, 1985 & $\mathrm{I}$ & $\mathrm{a}$ & $\mathrm{M}$ \\
\hline Necochea: Energía & * & $\mathrm{C}$ & $\mathrm{f}$ & $\mathrm{PI}$ \\
\hline Pergamino: Pergamino & Ricci et al., 2012, 2015 & $\mathrm{O}$ & $\mathrm{c}$ & $\mathrm{PO}$ \\
\hline Quilmes: Quilmes & * & $\mathrm{I}$ & $\mathrm{a}$ & $\mathrm{M}$ \\
\hline Quilmes: Bernal & * & $\mathrm{I}$ & $\mathrm{a}$ & $\mathrm{M}$ \\
\hline Saavedra: Pigüé & ** & $\mathrm{C}$ & $\mathrm{f}$ & TV \\
\hline Saladillo: Saladillo & Catalano et al., 2012; Ricci et al., 2012, 2015 & $\mathrm{O}$ & $\mathrm{e}$ & $\mathrm{PA}$ \\
\hline San Cayetano: San Cayetano & * & $\mathrm{C}$ & $\mathrm{f}$ & $\mathrm{PI}$ \\
\hline San Isidro: San Isidro & EcoRegistros & $\mathrm{I}$ & $\mathrm{a}$ & $\mathrm{D}$ \\
\hline San Miguel: Campo de Mayo & Di Iorio \& Turienzo, 2015 & $\mathrm{I}$ & $\mathrm{a}$ & $\mathrm{M}$ \\
\hline Tandil: Tandil & * & $\mathrm{C}$ & $\mathrm{f}$ & TV \\
\hline
\end{tabular}




\begin{tabular}{|l|l|c|c|c|}
\hline \multicolumn{1}{|c|}{ 8. Acromyrmex lundii } & \multicolumn{1}{|c|}{ Referencia bibliográfica } & C & ZA & SR \\
\hline Tapalqué: Tapalqué & \multicolumn{1}{|c|}{ Lacalidad } & G & e & PD \\
\hline Tigre: Delta & $*$ & IM & a & D \\
\hline Tigre: Isla Tres Bocas & Fernandez Valiela et al., 1954 & IM & a & D \\
\hline Tigre: Gral. Pacheco & EcoRegistros & IM & a & D \\
\hline Tornquist: Villa Ventana & $*$ & G & f & TV \\
\hline Tornquist: Chasicó & $*$ & G & f & TV \\
\hline Tres Arroyos: Tres Arroyos & Pompozzi, inéd. & C & f & PI \\
\hline $\begin{array}{l}\text { Vicente López: } \text { Reserva ecológica } \\
\text { Vicente López }\end{array}$ & $*$ & I & a & D \\
\hline Zárate: Zárate & EcoRegistros & IM & c & D \\
\hline
\end{tabular}

\begin{tabular}{|c|c|c|c|c|}
\hline \multicolumn{5}{|c|}{ 9. Acromyrmex rugosus } \\
\hline Partido: Localidad & Referencia bibliográfica & $\mathrm{C}$ & $\mathrm{ZA}$ & SR \\
\hline Pergamino: Pergamino & Ricci et al., 2012, 2015 & $\mathrm{O}$ & $\mathrm{c}$ & $\mathrm{PO}$ \\
\hline
\end{tabular}

\begin{tabular}{|c|c|c|c|c|}
\hline \multicolumn{5}{|c|}{ 10. Acromyrmex silvestrii } \\
\hline Partido: Localidad & Referencia bibliográfica & $\mathrm{C}$ & $\mathrm{ZA}$ & SR \\
\hline Cnel. Rosales: Bajo Hondo & Santschi, 1916 & $\mathrm{G}$ & $\mathrm{f}$ & $\mathrm{SP}$ \\
\hline Monte Hermoso: Monte Hermoso & Santschi, 1916 & $\mathrm{~T}$ & $f$ & TV \\
\hline Tornquist: Sierra de la Ventana & Bruch, 1916 & $\mathrm{G}$ & $\mathrm{f}$ & TV \\
\hline
\end{tabular}

\begin{tabular}{|c|c|c|c|c|}
\hline \multicolumn{5}{|c|}{ 11. Acromyrmex striatus } \\
\hline Partido: Localidad & Referencia bibliográfica & $\mathrm{C}$ & $\mathrm{ZA}$ & SR \\
\hline Bahía Blanca: Bahía Blanca & 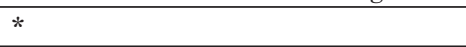 & IM & $\mathrm{f}$ & $\mathrm{SP}$ \\
\hline Guaminí: Laguna Guaminí & Montalti et. al, 2005 & $\mathrm{G}$ & $\mathrm{d}$ & $\mathrm{RL}$ \\
\hline Patagones: Carmen de Patagones & Mac Donagh, 1939** & $\mathrm{G}$ & $\mathrm{f}$ & $\mathrm{SP}$ \\
\hline San Isidro: San Isidro & Gallardo, 1916 b & $\mathrm{I}$ & $\mathrm{a}$ & $\mathrm{D}$ \\
\hline Villarino: Fortín Mercedes & * & $\mathrm{G}$ & $f$ & $\mathrm{SP}$ \\
\hline Villarino: Médanos & * & $\mathrm{G}$ & $\mathrm{f}$ & $\mathrm{SP}$ \\
\hline
\end{tabular}


